\title{
Numerical Evaluation of Derivatives of Functions
}

\author{
S. B. Sahoo ${ }^{1}$, M. Acharya ${ }^{2}$ and B. P. Acharya ${ }^{2}$ \\ ${ }^{1}$ Trident Academy of Creative Technology,BijuPatnaik University of Technology, \\ ${ }^{2}$ Institue of Technical Education \&Research,S'O'A University, Odisha(India) \\ ( ${ }^{1}$ sundarbadan@gmail.com, ${ }^{2}$ milu_acharya@gmail.com)
}

\begin{abstract}
An iterative technique based on the generalized Cauchy integral formula has been developed for the numerical evaluation of the derivatives of a real valued function $g(x)$ such that the function $z \mapsto g(z)$ is analytic in a domain which intersects the real axis. The transformed Gauss-Legendre rules meant for the numerical quadrature of analytic function along directed line segments has been employed for the computation of the derivatives.
\end{abstract}

AMS Subject Classification:primary 65D 25

Keywords:Derivative, analytic function, transformed Gauss-Legendre rules

\section{INTRODUCTION}

Numerical differentiation of a function is more difficult than numerical integration. This is because the later process requires only good continuity properties of the function, while for the former, the function is required to satisfy more complicated properties such as Lipschitzclasses. As a result of this, the process of numerical integration is relatively more stable than the process of numerical differentiation. However, there are many application in different branches of science and engineering and also in economics where the numerical evaluation of derivatives of a function becomes quite essential. The methods meant for the numerical differentiation of functions available in standardtextsare based on forward, backward and central differences or the theory of polynomial interpolation and Richardson extrapolation technique. These methods are sensitive to errors in the function values especially if these errors are not sufficiently small compared to the step size used in the differentiation formula.

In view of wide applications of numerical differentiation substantial research work has been undertaken for devising quite efficient methods. Some of the methods for numerical evaluation of derivatives of real as well as complex valued functions are due to Calio,Frontini and Milovanovic[1], Cullum[2], Hunter[3], Lyness and Moler[4], Micchelli [5], Tosic[6], and others.

Our aim in this paper is to formulate a method for the numerical evaluation of derivatives of a real valued function which is based on the generalized Cauchy integral formula of complex analysis. The method is an iterative one and makes use of the transformed Gauss-Legendre quadrature rules. It is noteworthy that the method can be extended for the numerical determination of the derivatives of complex valued analytic functions by suitably choosing the contour of integration in the generalized Cauchy integral formula.

\section{FORMULATION OF THE METHOD}

The generalized Cauchy integral formula concerning an analytic function $g(z)$ is statedbelow:

$$
g^{(n)}\left(z_{0}\right)=\frac{n !}{2 \pi i} \int_{\Gamma} \frac{g(z)}{\left(z-z_{0}\right)^{n+1}} d z
$$

where $z=x+i y, \Gamma$ is a closed contour lying inside the domain of analyticity of the function $g(z)$ surrounding the point $z_{0}$ and described in the positive sense.Let the contour $\Gamma$ be a square with vertices

$$
z_{j}=x_{0}+s(1+i) i^{j+1}, \quad j=1(1) 4
$$

and $x_{0}$ is a point on the $x$-axis where the values of the derivatives of the function $g(z)$ are sought and $s$ is a nonzero real numberwhich is small in magnitude such that the point $x_{0}$ is the centre of the square contour. Then setting $z_{0}=x_{0}$ in equation (1), we have the following: 


$$
g^{(n)}\left(x_{0}\right)=\frac{n !}{2 \pi i} \sum_{j=1}^{4} \int_{L_{j}} \frac{g(z)}{\left(z-x_{0}\right)^{n+1}} d z
$$

where $L_{j}$ is a directed line segment from the point $z_{j}$ to the point $z_{j+1}$ and $z_{5}=z_{1}$. The contour integral along $L_{j}$ under the summation sign in equation (3) which is denoted as $J_{j}$ can be represented in the following symmetric form:

$$
J_{j}=\int_{z_{0 j}-h_{j}}^{z_{0 j}+h_{j}} \frac{g(z)}{\left(z-x_{0}\right)^{n+1}} d z
$$

where $z_{0 j}=\left(z_{j+1}+z_{j}\right) / 2$ and $h_{j}=\left(z_{j+1}-z_{j}\right) / 2$.

For the numerical evaluation of the complex integral in the right hand side of equation (4) a number of methods have been devised (cf. Milovanovic [7]). From the point of view of accuracy amongst these methods the most preferable one is the transformed Gauss-Legendre rule proposed by Lether [8]. In general the m-point transformed Gauss-Legendre rule meant for the integral of an analytic function $\varphi(z)$ along a directed line segment from the point $z_{0}-H$ to $z_{0}+H$ is given by the following:

$$
\int_{z_{0}-h}^{z_{0}+h} \varphi(z) d z \approx H \sum_{k=1}^{m} c_{k} \varphi\left(z_{0}+H \eta_{k}\right)
$$

where $\eta_{k}$ 's are the zeros of the Legendre polynomial of degree $m$ and $c_{k}$ 's are the associated coefficients in the $m$-point Gauss-Legendre quadrature rule(cf. Abramowitz and Stegun[9]).

The first, second and third derivatives $(n=1,2,3)$ have been computed by applying the transformed GaussLegendre $m$-point rule given by equation (5). It is noteworthy that the central point $z_{0 j}$ of the path $L_{j}$ is nearest to the singularity $x_{0}$ if $m$ is odd. So for the purpose of computation of the integral $J_{j}$ we consider only even values of $m$ and set $m=12,14,16$. Taking $x_{0}=1.0, s=0.2$ the four integrals $J_{j}, j=1(1) 4$ in equation (3) have been computed replacing $\varphi(z) \operatorname{byg}(z) /\left(z-x_{0}\right)^{n+1}$ and assigning appropriate values to $z_{0}$ and $H$ in equation (5). Let the numerical approximations obtained for the $n^{\text {th }}$ derivatives $(n=1,2,3)$ for different functions $g(z)$ be denoted as $Q_{m}^{G L}\left(g^{(n)}\right)$ and the absolute errors be denoted as $\left|Q_{m}^{G L}\left(g^{(n)}\right)-g^{(n)}\right|$ whichare appended in Table-I.

Table-I

\begin{tabular}{|c|c|c|c|c|}
\hline$g(z)$ & $m$ & $\left|Q_{m}^{G L}\left(g^{(1)}\right)-g^{(1)}\right|$ & $\left|Q_{m}^{G L}\left(g^{(2)}\right)-g^{(2)}\right|$ & $\left|Q_{m}^{G L}\left(g^{(3)}\right)-g^{(3)}\right|$ \\
\hline \multirow{3}{*}{$\log ((1+z)$} & 14 & $1.56(\mathrm{e}-11)$ & $7.83(\mathrm{e}-12)$ & $7.85(\mathrm{e}-12)$ \\
\cline { 2 - 5 } & 16 & $4.62(\mathrm{e}-13)$ & $2.37(\mathrm{e}-13)$ & $1.96(\mathrm{e}-13)$ \\
\cline { 2 - 5 } & 20 & $4.44(\mathrm{e}-16)$ & $4.80(\mathrm{e}-15)$ & $2.83(\mathrm{e}-15)$ \\
\hline \multirow{3}{*}{$e^{z}$} & 14 & $8.52(\mathrm{e}-11)$ & $8.52(\mathrm{e}-11)$ & $8.52(\mathrm{e}-11)$ \\
\cline { 2 - 5 } & 16 & $2.51(\mathrm{e}-12)$ & $2.49(\mathrm{e}-12)$ & $2.37(\mathrm{e}-12)$ \\
\cline { 2 - 5 } & 20 & $2.66(\mathrm{e}-15)$ & $3.99(\mathrm{e}-15)$ & $1.63(\mathrm{e}-14)$ \\
\hline \multirow{3}{*}{$\sin (\mathrm{e}(\mathrm{z})$} & 14 & $1.69(\mathrm{e}-11)$ & $2.63(\mathrm{e}-11)$ & $5.26(\mathrm{e}-13)$ \\
\cline { 2 - 5 } & 16 & $4.99(\mathrm{e}-13)$ & $7.81(\mathrm{e}-13)$ & $1.12(\mathrm{e}-14)$ \\
\cline { 2 - 5 } & 20 & $4.44(\mathrm{e}-16)$ & $4.32(\mathrm{e}-15)$ & \\
\hline
\end{tabular}

\section{THE ITERATIVE SCHEME FOR DIFFERENTIATION}

In this section we consider first the numerical evaluation of the first derivative of the function $g(z)$ by making $n=1$ in equation (3)for the sake of simplicity.

For obtaining a fair accuracy of more than ten decimal places, $m$ (the number of nodes in the transformed Gauss-Legendre rule) has been assigned relatively large values in the previous section. To reduce the number of nodes the principal part arising due to the singularity $x_{0}$ (which is a pole of order two) should be subtracted out from the integrand given by equation (3) before the application of the transformed Gauss-Legendre rules. The principal part of the integrand when $n=1$ in the neighborhood of $x_{0}$ is given by

$$
p_{1}(z)=g\left(x_{0}\right)+\left(z-x_{0}\right) g^{(1)}\left(x_{0}\right) \text {. }
$$

Replacing the function $g(z)$ in the integral in equation (3) by $g(z)-p_{1}(z)$, we have the following equation: 


$$
\frac{1}{2 \pi i} \int_{\Gamma} \frac{g(z)}{\left(z-x_{0}\right)^{2}} d z=\frac{1}{2 \pi i} \int_{\Gamma} \frac{g(z)-p_{1}(z)}{\left(z-x_{0}\right)^{2}} d z+g^{(1)}\left(x_{0}\right)
$$

Decomposing the contour $\Gamma$ as $\bigcup_{j=1}^{4} L_{j}$ and applying the $m$-point transformed Gauss-Legendre rule to the integral in the right hand side of equation (7) we obtain the approximation for the integral in the left hand sideof (7) which represents the first derivative $g^{(1)}\left(x_{0}\right)$. Hence

$$
\begin{gathered}
g^{(1)}\left(x_{0}\right)=\frac{1}{2 \pi i} \int_{\Gamma} \frac{g(z)}{\left(z-x_{0}\right)^{2}} d z \\
\approx \frac{1}{2 \pi i} \sum_{j=1}^{4} h_{j} \sum_{\mu=1}^{m} \frac{c_{\mu}}{\left(\theta_{j \mu}-x_{0}\right)^{2}}\left\{g\left(\theta_{j \mu}\right)-g\left(x_{0}\right)-\left(\theta_{j \mu}-x_{0}\right) g^{(1)}\left(x_{0}\right)\right\}+g^{(1)}\left(x_{0}\right)
\end{gathered}
$$

where $\theta_{j \mu}=z_{0 j}+h_{j} \eta_{\mu}$.Denoting the unknown quantity $g^{(1)}\left(x_{0}\right)$ appearing in the right hand side of the approximate equation $(8)$ as $D^{(1)}(v)$ we have the following iteration scheme from the approximate equation (8).

$$
D^{(1)}(v+1)=\frac{1}{2 \pi i} \sum_{j=1}^{4} h_{j} \sum_{\mu=1}^{m} \frac{c_{\mu}}{\left(\theta_{j \mu}-x_{0}\right)^{2}}\left\{g\left(\theta_{j \mu}\right)-g\left(x_{0}\right)-\left(\theta_{j \mu}-x_{0}\right) D^{(1)}(v)\right\}+D^{(1)}(v)
$$

where $v=1,2,3, \ldots$ To commence the iteration process given by equation (9) a starting value for $D^{(1)}(1)$ is assigned as the initial guess for the first derivative $g^{(1)}\left(x_{0}\right)$ and the successive iterations are calculated until two consecutive iterates agree up to a desired number of decimal places. It is noteworthy that Acharya et al [10] have recently devised the technique for the numerical rectification of curves where the authors have computed the first derivative using the Cauchy integral formula and quadrature methods.

So far as the computation of the $n^{\text {th }}$ derivative $g^{(n)}\left(x_{0}\right)$ is concerned the iteration scheme can be constructed in the same vein as in case of the first derivative by subtracting out the following principal part from $g(z)$ in the numerator of the integrand given by equation (3).

$$
p_{n}(z)=\sum_{\rho=0}^{n} \frac{g^{(\rho)}\left(x_{0}\right)}{\rho !}\left(z-x_{0}\right)^{\rho}
$$

This leads to the following iteration scheme:

$$
=\frac{1}{2 \pi i} \sum_{j=1}^{4} h_{j} \sum_{\mu=1}^{m} \frac{c_{\mu}}{\left(\theta_{j \mu}-x_{0}\right)^{n+1}}\left\{g\left(\theta_{j \mu}\right)-\sum_{\rho=0}^{n-1} \frac{g^{(\rho)}\left(x_{0}\right)}{\rho !}\left(\theta_{j \mu}-x_{0}\right)^{\rho}-\frac{D^{(n)}(v)}{n !}\left(\theta_{j \mu}-x_{0}\right)^{n}\right\}+D^{(n)}(v)
$$

It is noteworthy that for the computation of the $n^{\text {th }}$ derivative the derivatives of orders $1,2, \ldots, n-1$ are already computed.

Setting $x_{0}=1$ and $s=0.2$ the derivatives of orders 1,2 and 3 have been computed using the scheme given by equation (11) employing the transformed Gauss-Legendre rules involving 2 and 4 nodes. The computed values of the absolute errors have been appended in Table-II. The quantity $v$ in table-II represents the minimum number of iterations needed for achieving the desired accuracy.

Table:-II

\begin{tabular}{|c|c|c|c|c|c|c|c|}
\hline \multirow{2}{*}{$g(z)$} & \multirow{2}{*}{$m$} & \multicolumn{3}{|c|}{$1^{\text {st }}$ derivative } & \multicolumn{2}{|c|}{$2^{\text {nd }}$ derivative } & \multicolumn{2}{c|}{$3^{\text {rd }}$ derivative } \\
\cline { 3 - 8 } & & $v$ & $\left|D^{(1)}(v)-g^{(1)}\right|$ & $v$ & $\left|D(v)-g^{(2)}\right|$ & $v$ & $\left|D(v)-g^{(3)}\right|$ \\
\hline \multirow{3}{*}{$\log (1+z)$} & 2 & 7 & $5.94(\mathrm{e}-009)$ & 7 & $3.67(\mathrm{e}-009)$ & 7 & $3.26(\mathrm{e}-008)$ \\
\cline { 2 - 8 } & 4 & 6 & $4.25(\mathrm{e}-014)$ & 6 & $3.89(\mathrm{e}-014)$ & 6 & $2.23(\mathrm{e}-012)$ \\
\hline \multirow{3}{*}{$e^{z}$} & 2 & 10 & $6.26(\mathrm{e}-011)$ & 9 & $5.84(\mathrm{e}-011)$ & 10 & $5.43(\mathrm{e}-012)$ \\
\cline { 2 - 8 } & 4 & 7 & $4.44(\mathrm{e}-016)$ & 7 & $4.44(\mathrm{e}-016)$ & 6 & $5.19(\mathrm{e}-014)$ \\
\hline \multirow{2}{*}{$\sin (\mathrm{z})$} & 2 & 9 & $2.12(\mathrm{e}-011)$ & 10 & $4.39(\mathrm{e}-012)$ & 11 & $6.94(\mathrm{e}-013)$ \\
\cline { 2 - 8 } & 4 & 7 & $1.16(\mathrm{e}-016)$ & 7 & $5.55(\mathrm{e}-016)$ & 6 & $1.35(\mathrm{e}-014)$ \\
\hline
\end{tabular}

It is noted that the highest accuracy of fifteen decimal places can be reached by using four point rule in maximum seven iterations. The technique is a simple one and is capable of yielding output of high order 
accuracy. Though computations have been made up to the third order derivative the method can be used for computation of derivatives of order more than three. It is further noteworthy that the iterative technique can be employed for evaluation of the derivatives of analytic functions of complex variables by a suitable choice of the contour $\Gamma$.

\section{REFERENCES}

[1] Calio, F., Frontini, M. and Milovanovic , G.V., Numerical differentiation of analytic functions using quadratures on semicircle, Comptu. Math. Appl., 22, 1991, 99-106.

[2] Cullum, J., Numerical differentiation and regularization, SIAM J. Numer. Anal., 8, 1971,254-265.

[3] Hunter, D. B., An iterative method of numerical differentiation,Comput. J., 3, 1960, 270-271.

[4] Lyness, J. N.; Moler, C. B., "Numerical differentiation of analytic functions", SIAMJ.Numer. Anal. 4,1967, $202-210$.

[5] Micchelli, C. A., On an optimal method for the numerical differentiation of smooth functions, J. Approx. Theory, 18, 1976, 189-204.

[6] Tosic D.D. , Numerical differentiation of analytic functions,Univ. Beog. Publ. Elektrotehn. Fak. Ser. Mat. Fiz. No 678- No715, 1980, $175-182$.

[7] Milovanovic, G.V., Generalized quadrature formulae for analytic functions, Appl. Math. \& computation, 218, $2012,8537-8551$.

[8] Lether, F. G., OnBirkhoff- Young quadrature of analytic functions, J. Comp. Appl. Math. 2, 1976, 81-84.

[9] AbramoiwitzM. andStegun I. A., Handbook of mathematical functions, Nat. Bur. Stand. Appl. Math., Ser. No.55, US Govt. Printing Office, 1964

[10] Acharya, B. P., Acharya, M. and Sahoo, S. B., Numerical Rectification of Curves, Applied Mathematical Sciences, Vol. 8 ( 17), 2014, $823-828$ 